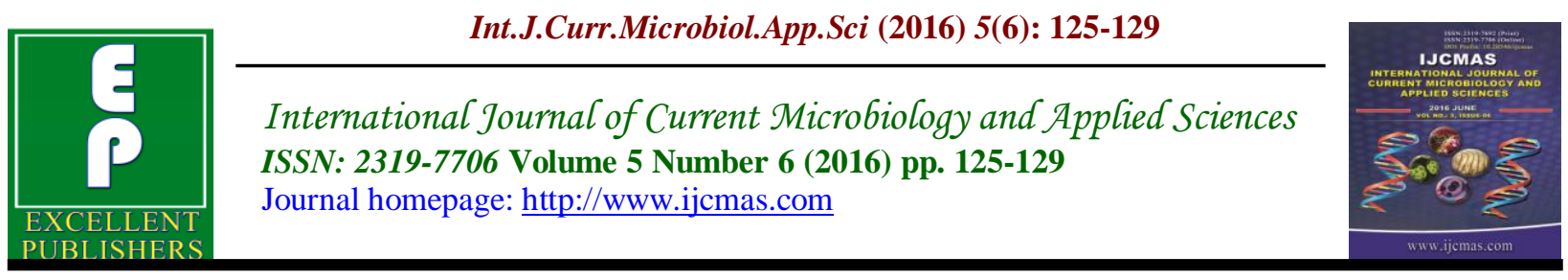

Original Research Article

http://dx.doi.org/10.20546/ijcmas.2016.506.015

\title{
Evaluation of a Cold Staining Method for Detecting Acid Fast Bacilli in the Sputum
}

\author{
Kiran Yadav*, Gunjan Dutta and Jugal Kishor Aggarwal \\ Saraswathi Institute of Medical SciencesHapur, Uttar Pradesh, India \\ *Corresponding author
}

\begin{abstract}
A B S T R A C T
Keywords

Acid fast bacilli,

Cold stain,

Tuberculosis,

Ziehl Nelseen

stain.

Article Info

Accepted:

11 May 2016

Available Online:

10 June 2016

Microscopic examination of sputum is the cornerstone in diagnosis of pulmonary tuberculosis in developing world, which usually done by Ziehl-Neelsen (ZN) method. However, due to limited laboratory facilities this procedure appears to be adversely affected in field conditions and at peripheral health institutions. Hence, it has become necessary to look for a procedure, which can be used as alternative in such conditions. We evaluated cold staining method for detection of acid fast bacilli in sputum smears. Total 587consecutive sputum samples both spot and early morning from 300 patients were collected and were stained with both Z-N stain and cold AFB stain and results were compared. Out of 300 patients, a total of 58 (19.3\%) were AFB positive by Z-N stain and 55 (18.3\%) were positive by cold staining method. By comparing the two we get sensitivity of $94.83 \%$, Specificity of $100 \%$; Positive predictive value of $100 \%$, and Negative predictive value of $98.78 \%$ by cold staining method against Z-N stain.
\end{abstract}

\section{Introduction}

Tuberculosis is a disease of great importance in developing countries, such as India, where it has caused considerable morbidity and mortality. Sputum smear microscopy is the cornerstone in diagnosis of pulmonary tuberculosis, which is usually done using Ziehl-Neelsen (ZN) method (Vasanthakumari et al., 1986; Gupta et al., 2009; Chandrasekaran et al., 1991). The use of ZN Method in remote areas of our country, however, suffers from operational limitations due to shortage of properly trained and experienced laboratory technicians in peripheral centres, and non availability, or short supply of spirit, essential for heating carbol fuchsin. Here we evaluate Cold Staining (CS) as an alternative test to $\mathrm{ZN}$. CS is one modification of $\mathrm{ZN}$ which requires carbol fuchsine as a primary stain and Gabbet's methylene blue, both as a decolorizer and counter stain. Compared to ZN; CS is simple and easy to learn and practice, it is economical and less cumbersome, is suitable under field conditions, and can be practiced even in remote areas and at periphery where laboratory facilities are limited. There are also more practical advantages of CS like, no need of heating in the staining procedure and eliminated the need for separate decolorizing step (requires only two 
reagents in the staining procedure) (Gokhale et al., 1990).

\section{Materials and Methods}

587 sputum samples 300 spot and 287 early morning samples were collected from 300 patients suspected of having pulmonary tuberculosis at Saraswathi Institute of Medical Sciences, Hapur, India. The smears of each sample were prepared in duplicate; one each for $\mathrm{ZN}$ and CS. For the preparation of smears to be submitted to stains, samples of each specimen were air-dried and heatfixed.

\section{Z-N Method}

One set was stained by the standard- ZN method, in which $1 \%$ carbol fuchsin was used. The RNTCP guidelines were followed to stain the smears. The air-dried smear slides were fixed over a flame three to five times for 3 to $4 \mathrm{~s}$. The slides were then placed on a staining rack, and filtered carbol fuchsin was poured to cover the entire slide. The slides were heated from underneath for 5 min until vapors started rising. After 5 min, slides were gently rinsed with tap water to remove the excess carbol fuchsin stain. The smears were decolorized with $25 \%$ sulfuric acid for 2 to $4 \mathrm{~min}$ and again rinsed with water. The slides were counterstained for 30 to $60 \mathrm{~s}$ using $0.1 \%$ methylene blue solution. The slides were rinsed with tap water, allowed to dry, and examined using oil immersion objective (100x). The composition of the reagents was as follows, $1 \%$ carbol-fuchsin was prepared with $1 \mathrm{gm}$ of basic fuchsin dissolved in $50 \mathrm{ml}$ molten phenol; $100 \mathrm{ml}$ of ethanol (95\%) was added to the fuchsin - phenol mixture. The solution was diluted with distilled water to make a volume of $1,000 \mathrm{ml}$ then it was filtered. Decolorizing agent sulfuric acid $(25 \%)$ was prepared with $250 \mathrm{ml}$ concentrated sulfuric acid which was slowly added to $750 \mathrm{ml}$ distilled water. Methylene blue $(0.1 \%)$ was prepared with 1 gram methylene blue dissolved in $100 \mathrm{ml}$ distilled water.

\section{CS}

For the preparation of smears to be submitted to CS, samples of each specimen were air-dried and heat-fixed. The slides were then flooded with $1 \%$ carbol-fuchsin stain $(10 \mathrm{~g}$ of basic fuchsin, $100 \mathrm{~mL}$ of methylated spirit, and $50 \mathrm{~g}$ of phenol and distilled water was added to make a final volume of $1,000 \mathrm{~mL}$ ). This was allowed to stand at room temperature for $10 \mathrm{~min}$. The smears were then washed in running water and counterstained with Gabbett's methylene blue (1 $\mathrm{g}$ of methylene blue, 20 $\mathrm{mL}$ of sulfuric acid, $30 \mathrm{~mL}$ of absolute alcohol, and $50 \mathrm{~mL}$ of distilled water) for 2 min. The slides were then washed, air-dried and examined using oil immersion objective $(100 \mathrm{x})$. At least 300 fields were observed by two experienced observers before giving negative and the slides were graded as per RNTCP (http://www.tbcindia.org/pdfs ., 2005).

\section{Results and Discussion}

A total of 587 sputum samples both spot and early morning were obtained from 300 patients, spot samples were obtained from all the patients, however early morning samples could be obtained from 287 patients only since some of the patients did not come back with morning samples. In our study $194(64.67 \%)$ patients were males and 106 (35.3\%) females. Male preponderance was noted in patients presenting with symptoms of pulmonary tuberculosis.

Of the total, $58(19.3 \%)$ had proven tuberculosis by positive AFB microscopy 
using the $\mathrm{ZN}$ method. On the other hand, among the 300 patients with suspected case of pulmonary tuberculosis, 55 (18.3\%) were positive for AFB by the cold staining method. All those smears which were positive by cold AFB method were also positive by Z-N method.

There were 94 AFB positive samples from 58 patients out of 300 patients who were found to be AFB positive by any of the methods in at least one of the two samples sputum submitted for analysis (Table no. 1). Out of these 03 patients were completely missed by cold AFB method, most of these were either scanty or grade $1+$ positive. There were no samples or patients which were detected positive by cold AFB staining alone.

Tuberculosis is a major public health problem, and its control has become a challenge in developing countries, such as India (Rao., 2007). Case finding is quite important for the control of the disease and is chiefly achieved by sputum smear microscopy. Throughout the country in all Primary health centers sputum smear microscopic examination successfully implemented by the RNTCP. Applicability of the Ziehl-Nelseen technique appears to be adversely affected, especially at peripheral health institutions, due to limited laboratory facilities that can satisfy the procedure. Hence, the present study aimed to evaluate cold stain method which can be used as alternative in such conditions.

In the present study, the interesting finding is that the CS Method has not shown any positive result even on a single specimen which was not positive by the $\mathrm{ZN}$ method. On the other hand, there were three patients negative for AFB by CS method. These two observations suggest that CS Method is at least as specific as $\mathrm{ZN}$ Method although somewhat less sensitive.

The Z-N was superior to the Gabbets cold staining method in our study but there was a good agreement between them. The tubercle bacilli morphology in the Gabbets staining method appear more delicate and closer to their morphology but are also fainter of than those seen with the Z-N stain, which may be reason for the false negative results compared by the $\mathrm{Z}-\mathrm{N}$ method. In the conventional Ziehl-Neelsen method there is a better penetration of stain through the complex cell surface structure due to the heating therefore the organism appears brighter against background.

Although it is difficult to conclude, since none of the specimens could be cultured for want of facilities, the $\mathrm{ZN}$ could give a few false positive results. Similar observation was seen by other scholars too (Gokhale et al., 1990; Pandey et al., 2009).

Table.1 Findings of Z-N staining and Cold AFB staining

\begin{tabular}{|l|c|c|}
\hline Particulars & Z-N Staining & Cold AFB staining \\
\hline AFB Positive Samples & 94 & 86 \\
\hline AFB Negative Samples & 493 & 501 \\
\hline Total & 587 & 587 \\
\hline
\end{tabular}


Table.2 Overall comparison of Cold stain method with the Ziehl-Neelsen method

\begin{tabular}{|l|l|l|l|}
\hline \multicolumn{5}{|c|}{ ZN } \\
\hline Report & Positive & Negative & Total \\
\hline Positive CS & 55 & 0 & 55 \\
\hline Negative & 03 & 242 & 245 \\
\hline Total & 58 & 242 & 300 \\
\hline
\end{tabular}

ZN: Ziehl-Neelsen; CS: Cold stain;

Positive $=$ any positive $($ scanty, $1+, 2+$ and $3+) ;$ Negative $=$ no acid fast bacilli per oil immersion in at least 100 fields; Sensitivity $=94.83 \%$; Specificity $=100 \%$; Positive predictive value $=100 \%$; Negative predictive value $=$ $98.78 \%$;

The Gabbet's method describes carbol fuchsin longer exposure helps the uniform penetration of the dye through the cell wall, the staining solution concentration is same as that used in the Z-N method and no extra cost is involved. The decolorizer used in the Gabbett's methylene blue includes both acid and alcohol, making it superior to the conventionally used only acid as decolorizer in the hot method (Sonnerwirth, 1980; Mackie and McCartney, 1989). This is useful as it eliminates the false positive smears due to the non pathogenic and contaminant mycobacteria which are only acid fast. These are occasionally recovered from urine samples and tap water but are of no clinical significance.

In this study, we found that the CS method has the advantages of being highly sensitive, economical and easy to learn and simple to perform as compared to the $\mathrm{ZN}$ stain method. Similar observations were also seen by many other authors (Tripathi et al., 2001; Deshmukh et al., 1996). To conclude, the Cold staining method is an improvement over the $\mathrm{Z} \mathrm{N}$ stain. Cold stain method can be used in routine for staining sputum sample especially in settings with high workloads or remote areas due to its high sensitivity, simplicity and rapidity.

\section{References}

Chandrasekaran, S., Sreenivas, T.R., Chaudhuri, K. 1991. Modified sputum microscopy techniques sparing the use of alcohol for field application. Indian J. Tub., 38: 8790.

Deshmukh, S.R., Sarita, B.M., Kendra, P.B., Nagoba, B.S. 1996. A Comparision of Sputum Examination for AFB. Ind. J. Med. Res., 103: 294-295.

Gokhale, S., Qadir, S., Nagra, J.S., Chakraborty, A.K. 1990. Efficiency of cold staining.

method of AFB in sputum a comparison with ziehl neelsen method under field conditions. Indian J. Tub., 37: 135-137.

Gupta, S., Prasad, V., Bairy, I., Muralidharan, S. 2009. Comparative evaluation of two cold staining methods with the ziehl-neelsen method for the diagnosis of tuberculosis. Southeast Asian J. Trop. Med. public health., 40: 765769

http://www.tbcindia.org/pdfs/Module\%20for \%20Laboratory\%20Technician. pdf 2005, 23-24

Mackie, Mc Cartney. 1989. Practical Medical Microbiology. 13th ed.Churchill Livingstone Longman Group UK limited pp. 46 - 48.

Pandey, A., Madan, M., Asthana, A.K., Kansal, R., Das, A. 2009. Cold acid fast staining method: Efficacy in diagnosis of Mycobacterium tuberculosis. Afr. J. Microbiol. Res., 
3: 546-549.

Rao, V.G. 2007. Tuberculosis control: Current status and challenges. RMRCT Update, 4(2) : 1-4.

Sonnerwirth, A.C. In: Sonnerwirth AC, Javett Leonard. 1980. Editor Gradwohl's clinical laboratory method and diagnosis, 2(8) ed, London: The CV Mosby company: pp. $1385-1388$.
Tripathi, D.G., Desai, M.W., Mesquita, A.M. 2001. Evaluation of a novel, two components, two step AFB cold staining method. Ind. J. Med. Microbiol., 19(3): 163-165.

Vasanthakumari, R., Jagannath, K., Rajasekaran, S. 1986. A cold staining method for acid-fast bacilli. Bull. World Health Organ., 64: 741743.

\section{How to cite this article:}

Kiran Yadav, Gunjan Dutta and Jugal Kishor Aggarwal. 2016. Evaluation of a Cold Staining Method for Detecting Acid Fast Bacilli in the Sputum. Int.J.Curr.Microbiol.App.Sci. 5(6): 125129. doi: http://dx.doi.org/10.20546/ijcmas.2016.506.015 